\title{
Microbiological Profile in Endodontic-periodontal Lesion
}

\author{
${ }^{1}$ Meenakshisundaram Rajasekaran, ${ }^{2}$ Deepavalli Arumuga Nainar, ${ }^{3}$ Swarna Alamelu, ${ }^{4}$ Arun KV
}

\begin{abstract}
Endodontic-periodontal lesion is a clinical manifestation of the pathologic intercommunication between pulpal and periodontal tissues. In general, these lesions are multifaceted in nature and can have a varied pathogenesis. Fundamental to our understanding of the pulpoperiodontal lesions is the key role of the pathogenic ecosystem that exists within this complex structure. Evidence suggests that there exists a wide range of microbial species in the periapical and periodontal tissues and that their concurrent pathogenesis is constantly linked to the bacterial interrelations between the two tissue types. Understanding the microbial involvement is of utmost significance as it offers a rationale for differential diagnosis and subsequent management of such lesions. This review has attempted to provide a basic insight on the microbiome or the microbial flora entailed in the bidirectional pathogenesis of endodontic-periodontal lesions.
\end{abstract}

Keywords: Endodontic-periodontal lesion, Microbiology, Periapical lesion, Periodontal disease, Persistent disease.

How to cite this article: Rajasekaran M, Nainar DA, Alamelu S, KV Arun. Microbiological Profile in Endodontic-periodontal Lesion. J Oper Dent Endod 2016;1(1):25-29.

\section{Source of support: Nil}

\section{Conflict of interest: None}

\section{INTRODUCTION}

The correlation between pulpal and periodontal disease was first described by Simring and Goldberg in $1964 .^{1}$ Ever since, the term 'endodontic-periodontal lesion' has been a matter of speculation with regard to its microbial entity which is either distinct or akin to both tissue types. The pulp and the periodontium are envisioned as a single biologic unit which is ectomesenchymal in origin. ${ }^{2}$ Such common embryonic development leads to their anatomic associations and, hence, a lasting functional relationship in health and disease. The microbial etiology controls and regulates the clinical course of the disease and,

\footnotetext{
${ }^{1,4}$ Professor, ${ }^{2}$ Lecturer, ${ }^{3}$ Reader

${ }^{1}$ Department of Conservative Dentistry and Endodontics, Ragas Dental College and Hospital, Chennai, Tamil Nadu, India

${ }^{2-4}$ Department of Periodontics, Ragas Dental College and Hospital, Chennai, Tamil Nadu, India

Corresponding Author: Meenakshisundaram Rajasekaran Professor, Department of Conservative Dentistry and Endodontics Ragas Dental College and Hospital, Uthandi-600119, Chennai Tamil Nadu, India, e-mail: endoraja@yahoo.com
}

consequently, its treatment planning. ${ }^{3}$ Numerous studies have investigated the influence of periodontal disease on pulpal pathosis microbiologically and vice vers $a^{4,5}$ while there are others who have shown unique species in the two lesions. However, there were many limitations to these studies in the methodologies that have been used, in the type of samples taken and the state of the disease process and, therefore, could not arrive at definitive conclusions. So, a larger understanding of the association of the microbial flora in the infected root canal and the periodontal pocket in combined pulpoperiodontal lesions will prove useful in determining the etiology of this complex disease process.

Hence, this review attempts at broadening the scope of understanding the microbiological profile in the endodontic-periodontal lesions.

The microbial community disseminate through various pathways of communication. These include the anatomical and nonphysiological pathways. ${ }^{3}$ The anatomical pathways are vascular passages which comprises of the apical foramen, accessory canals and the dentinal tubules. The apical foramen is the primary means of connection between the pulp and the periodontium. ${ }^{6}$ Periodontal disease can cause pulpal necrosis predominantly after progressing apically and involving the apical foramen. Various bacterial products initiate and mount an inflammatory response thereafter. This in turn results in further periodontal tissue destruction. The lateral and the accessory canals are present all along the root surface and more so in the apical third. They usually contain connective tissue and blood vessels which connect the circulatory system of the pulpoperiodontal complex. Studies have shown that these canals are potential pathways for spread of microbial and inflammatory products between the pulp and the periodontium. ${ }^{7}$ Studies have also stated that the periodontal endodontic problems were much more frequent in the molars than in the anterior teeth because of the greater number of accessory canals present in the molars. ${ }^{8}$ Another anatomical pathway of communication is the presence dentinal tubules when they get exposed in areas of denuded cementum. ${ }^{9}$ Apart from these anatomical pathways, studies indicate that the extent and severity of palatogingival grooves are also associated with combined incidence of periodontal disease with pulpal pathosis. ${ }^{10}$ The nonphysiological pathways include iatrogenic root canal perforations due to improper 
manipulation of endodontic instruments and vertical root fractures caused by trauma. ${ }^{3}$ Through these pathways, the organisms can disseminate from the infected pulp to the healthy periodontium or from periodontal disease sites to vital pulp.

\section{ENDODONTIC AND PERIODONTAL MICROBIOLOGY}

The infected root canal is initially composed of a polymicrobial flora, but, with time, allows only a restricted group of microbes to thrive among the entire oral flora. Oxygen is one of the important ecological determinants in this species specific environment. ${ }^{11}$ The pioneer species consume the oxygen, lowers the redox potential and makes the environment predominantly anaerobic in nature. Certain precise mechanisms facilitate the survival of obligate anaerobes which include the synergistic behavior of the endodontic organisms along with the interplay of a number of factors like the availability of potential nutrients, production of growth factors and enzymes, inhibition of phagocytosis, reduction in oxygen concentration and, hence, the reduced redox potential. ${ }^{12}$

Bacteria in the root canal do not grow as separate colonies instead form interconnected communities as a biofilm within an extracellular matrix. Synergistic bacteria become strongly associated according to the type of nutrients in the environment, viz. Fusobacterium nucleatum is strongly associated with Peptostreptococcus micros, Porphyromonas endodontalis and Campylobacter rectus; Prevotella intermedia with P. micros and Peptostreptococcus anaerobius, P. anaerobius with eubacteria, etc. In common, species of prevotella, eubacteria and peptostreptococcus are positively correlated with one another in an endodontic environment. ${ }^{13}$ All these organisms exhibit a common property that they are anaerobic and ferment amino acids and peptides. ${ }^{14}$

However, studies also show association of 'specific pathogens' in endodontic lesions. Porphyromonas endodontalis and $P$. gingivalis most commonly associated with endodontic infections; P. nigrescens and Mitsuokella dentalis are often being isolated from endodontic samples. Certain specific microbial groups, like Actinomyces, Staphylococcus aureus and Enterococcus faecalis, are commonly associated with refractory endodontic lesions. $^{15}$

\section{MICROBIAL PROFILE OF CANALS WITH PERSISTENT INFECTION}

Evidence from various studies suggests that there is high prevalence of enterococci and streptococci in persistent endodontic lesions in the root canal filled teeth. ${ }^{16}$ Enterococcus faecalis is known to be an important opportunistic pathogen found in persistent apical periodontitis. ${ }^{17}$ The other species found in greater fractions include Actinomyces species, peptostreptococci, ${ }^{18}$ Pseudoramibacter alactolyticus, Filifactor alocis, Propionibacterium propionicum and Candida albicans. ${ }^{19}$

\section{What leads to the Persistence of These Organisms in the Root Canal?}

By and large, the persistence of infection could be owing to imprecision during endodontic procedures like poor asepsis, improper access cavity design, inadequate instrumentation and neglected canals. In such instances, the microbial flora is almost similar to that present in untreated root canals. Sometimes even in properly filled canals with intact coronal seal, bacteria if persistent, should have either escaped the antimicrobial treatment or have entered during treatment and found the place conducive for thriving due to presence of transudate like fluid from the periapical tissues. ${ }^{20}$

The three strategies which have been proposed as evasive mechanisms employed by the microbes are sequestration, cellular evasion and humoral evasion. ${ }^{21}$ In sequestration, there is formation of a physical barrier between the microbe and the host, while in the immune response evasion, it negates killing by the leukocytes, antibody secretion and complement activation.

Studies have shown E. faecalis and Candida species to remain sequestered in the canal ramifications and in dentinal tubules and remain protected against the antimicrobial mechanisms. In addition, endurance of starvation or nutrient depletion is yet another unique trait of E. faecalis which makes it a predominant organism in persistent infections. ${ }^{22}$

\section{Similarities and Differences in the Microbial Profile of the Endodontic-periodontal Milieu}

One of the most contemplated areas of endodontic microbiology has been to ascertain how microorganisms of the periodontium and endodontium link together. Various traditional and molecular methods have been utilized to analyze the connection between endodontic and periodontal niches with respect to oral microbiome.

Gomes et al demonstrated the presence of the red complex organisms namely $T$. forsythia, Porphyromonas gingivalis and Treponema denticola in the periradicular region and found to be associated with the etiology of periradicular diseases. ${ }^{23}$ Rocas also identified red complex organisms within infected root canals and found that at least one member was found in $66 \%$ of the cases. ${ }^{24}$

Rupf et al employed specific PCR methods to identify Aggregatibacter actinomycetemcomitans, Tannerella forsythia, Eikenella corrodens, F. nucleatum, P. gingivalis, 
P. intermedia, and Treponema denticola. These organisms were identified in all samples from endodontic infection sites and were also found in sites with chronic periodontitis. They concluded that periodontopathogens habitually accompany endodontic lesions and supported the proposal that endodontic-periodontal interrelationships are a critical pathway for both diseases. ${ }^{25}$

In yet another study, Hong Li et al compared the microbial flora in the root canal and from the adjacent periodontal pocket; using PCR-based denaturing gradient gel electrophoresis (PCR-DGGE). Totally 60 species were analyzed by sequencing out of which 43 species were common to both root canal and pocket samples. The most predominant of all bacteria was Filifactor alocis, followed by Parvimonas micra, Porphyromonas gingivalis, and Tannerella forsythia. Most of the microflora in the root canal samples resembled that of the periodontal pocket. The similarity in microbial flora between the root canal and the pocket suggested that the contents of the pocket could be a possible source of root canal infection. ${ }^{26}$

Although there are quite a number of studies which have suggested similarities, combined endodonticperiodontal lesion in addition, harbor diverse and complex bacterial communities. Kurihara et al observed a significant difference in the microbiological aspects of endoperiodontal lesions from root canal contents and from the deepest portion of the periodontal pocket, while the periodontal pocket displayed a large variety of species including the spirochetes, root canal was restricted to Gram-positive cocci, including Peptostreptococcus and Streptococcus, or Gram-positive rods, such as Actinomyces. ${ }^{5}$

Aggregatibacter actinomycetemcomitans was not found to be a relevant endodontic pathogen as the microenvironment of the root canal does not favor the survival of this pathogen as that of the periodontal pocket. This variation results in low occurrence of these bacteria in the infected root canals. ${ }^{24}$

\section{DISCUSSION}

An endoperiodontic lesion may be defined as degenerative involvement of pulp and periodontium subsequently leading to pulpal necrosis and formation of clinical periodontal pocket in the same tooth. Studies suggest, dental pulp has the ability to withstand considerable insults, unless periodontal disease progresses apically and reach the root apex and initiate pulpal inflammation.

Specific bacterial colonies associate each other and form interconnected communities within an intracellular matrix. Such a description was reported by Nair who described them as coaggregating communities with a palisade structure. ${ }^{27}$ The bacteria in such a community were relatively protected than the planktonic ones and are more resistant to antimicrobial treatment measure. ${ }^{28}$ However, only limited information is available on its physiology and growth, and thus paves way for future research.

Although there are similarities in the microbial flora existing in the true combined lesions and those that exist in primary endodontic and primary periodontal lesions, studies have shown that there are distinct microflora in the combined endoperiodontal lesion that are either completely absent or present in very negligible amounts in either the endodontic or periodontal lesions. Johnson et al analyzed the proteolytic activity of Bacillus pumilus isolated from combined endodontic-periodontal lesion. ${ }^{29}$ This species showed extracellular activities degrading elastin, collagen and other substrates. Such a virulence factor results in tissue damage in endodontic-periodontal lesion.

Furthermore, bacterial analysis of combined endodontic-periodontal lesions by polymerase chain reaction denaturing gradient gel electrophoresis ${ }^{30}$ showed a complicated yet similar bacterial profile in periodontal pocket and root canal ranging from 13.1 to $62.5 \%$ indicating an overlap in the bacterial profile. Campylobacter, Fusobacterium, Neisseria, Peptostreptococcus, Veillonella, Aggregatibacter, enterobacter and haemophilus in dental plaque and Mogibacterium, Cornybacterium, Neisseria and Actinomyces in necrotic pulp were found. Moreover, A. odontolyticus was detected in necrotic pulp and may be a potential pathogen in combined lesion. The study also concluded that plaque samples showed more bacterial microflora as the periodontium is more exposed to oral environment and that there were new bacterial species detected in both the root canal and the periodontal pocket of a combined lesion.

In addition, clonal analysis of anaerobic bacteria associated with endodontic-periodontallesions ${ }^{31}$ suggested that $P$. intermedia was the most prevailing species of the colonies in periodontal pockets, whereby $P$. gingivalis and $P$. intermedia were most evidently found in the root canals. Besides, there was simultaneous colonization of the pulp and the periodontal microenvironment by $P$. gingivalis, $T$. forsythia and $P$. endodontalis. The study concluded that different clones of $P$. intermedia and $P$. gingivalis were found to colonize similar anatomic sites in endodontic-periodontal lesions.

Lastly, human herpes viruses in gingival tissue were associated with increased incidence of $P$. gingivalis, $P$. nigrescens, $T$. denticola, $P$. intermedia, and $A$. actinomycetemcomitans, proposing that they may play a role in promoting overgrowth of these periodontal pathogens ${ }^{32}$ and this in turn could affect the pulpal pathosis. The reason that periapical lesions sometimes show quiescence for long period of time could be 
attributed to the absence of herpes virus infection or viral inactivation in the associated lesion.

It is apparent from the above-mentioned studies that there is preponderance of periodontopathogens in the periapical sites both in the primary endodontic lesions as well as true combined lesions. However, the survival of endodontic pathogens is restricted in the periodontal disease sites. The basis for such a difference in existence is explained by various phenomena. Firstly, the periodontal pathogens slowly progress apically along with formation of periodontal pocket and are able to thrive well in suitable microenvironment forming microbial coaggregations till it reaches the apex and initiates periapical infection. This favorable environment is provided by increased production of gingival crevicular fluid, pronounced bleeding, proteolysis and increased hemin levels, i.e. a 'nutrient rich' zone on the whole. On the other hand, the endodontic microflora which is predominantly anaerobic, when progressing coronally gets introduced to a microaerophilic environment arresting further growth.

Based on the literature evaluated, it can be comprehended that the endodontic and periodontal milieu share numerous similarities with respect to its microbial flora and that cross contamination can occur through various pathways between the periodontium and the pulpal tissue. However, there seems to be a predominance of periodontal pathogens in primary endodontic lesions as well as true combined lesions.

\section{CONCLUSION}

Although there are large resemblances in the microbiological profile of endodontic and periodontal lesions, it could possibly be characterized by a distinct microbiome in its individual compartments, which is thought to be influenced by their respective inflammatory milieu and coaggregation patterns. So, the rationale for the use of chemotherapy as a 'stand alone' or as an adjuvant therapy does not hold ground any further. Mechanotherapy resulting in disruptions of the biofilm will continue to be the mainstay approach in the management of endodonticperiodontal lesion. In future, the endodontic-periodontal microbiome should be investigated as whole rather than individual microbes to study the extent of bacterial communication and thus to understand the pathogenesis of such lesions.

\section{REFERENCES}

1. Simring M, Goldberg M. The pulpal pocket approach: retrograde periodontitis. J Periodontol 1964;35:22-48.

2. Mandel E, Machtou P, Torabinejad M. Clinical diagnosis and treatment of endodontic and periodontal lesions. Quintessence Int 1993;24:135-139.
3. Zehnder M, Gold SI, Hasselgren G. Pathologic interactions in pulpal and periodontal tissues. J Clin Periodontol 2002;29: 663-671.

4. Kerekes K, Olsen I. Similarities in the microfloras of root canals and deep periodontal pockets. Endod Dent Traumatol 1990; 6:1-5.

5. Kurihara H, Kobayashi Y, Francisco IA, Isoshima O, Nagai A, Murayama Y. A microbiological and immunological study of endodontic-periodontic lesions. J Endod 1995;21:617-621.

6. Rotstein I, Simon JH. Diagnosis, prognosis and decisionmaking in the treatment of combined periodontal-endodontic lesions. Periodontol 2000 2004;34:165-203.

7. DeDeus QD. Frequency, location and direction of the lateral, secondary and accessory canals. J Endod 1975;1:361-366.

8. Lowman JV, Burke RS, Pelleu GB. Patent accessory canals: incidence in molar furcation region. Oral Surg Oral Med Oral Pathol 1973;36:580-584.

9. Harrington GW, Steiner DR. Periodontal-endodontic considerations. In: Walton RE, Torabinejad M, editors. Principles and practice of endodontics, 3rd ed. Philadelphia: WB Saunders Co; 2002. p. 466-484.

10. Everett FG, Kramer GM. The distolingual groove in the maxillary lateral incisor; a potential hazard. J Periodontol 1972;43(6):352-361.

11. Loesche WJ, Gusberti F, Mettraux G, Higgins T, Syed S. Relationship between oxygen tension and subgingival bacterial flora in untreated human periodontal pockets. Infect Immun 1983;42:659-667.

12. Sundqvist G. Taxonomy, ecology and pathogenicity of the root canal flora. Oral Surg Oral Med Oral Pathol 1994 Oct; 78(4):522-530.

13. Sundqvist G. Associations between microbial species in dental root canal infections. Oral Microbiol Immunol 1992;7: 257-262.

14. Steeg PF, van der Hoeven JS. Development of periodontal microflora on human serum. Microb Ecol Health Dis 1989; 2:1-10.

15. Drucker DB, Natsiou I. Microbial ecology of the dental root canal. Microb Ecol Health Dis 2000;12:160-169.

16. Molander A, Reit C, Dahlen G, Kvist T. Microbiological status of root-filled teeth with apical periodontitis. Int Endod J 1998; 31:1-7.

17. Cheung GS, Ho MW. Microbial flora of root canal-treated teeth associated with asymptomatic periapical radiolucent lesions. Oral Microbiol Immunol 2001;16:332-337.

18. Hancock HH, Sigurdsson A, Trope M, Moiseiwitsch J. Bacteria isolated after unsuccessful endodontic treatment in a North American population. Oral Surg Oral Med Oral Pathol Oral Radiol Endod 2001;91:579-586.

19. Siqueira JF, Rocas IN. Polymerase chain reaction-based analysis of microorganisms associated with failed endodontic treatment. Oral Surg Oral Med Oral Pathol Oral Radiol Endod 2004;97:85-94.

20. Figdor D, Sundqvist G. A big role for the very smallunderstanding the endodontic microbial flora. Aust Dent J 2007;52(Suppl 1):S38-S51.

21. Nataro JP, Blaser MJ, Cunningham-Rundles S. Persistent bacterial infections: commensalism gone awry or adaptive niche? In: Nataro JP, Blaser MJ, Cunningham-Rundles S, editors. Persistent bacterial infections. Washington, DC: ASM Press; 2000. p. 3-10. 
22. Sedgley CM, Lennan SL, Appelbe OK. Survival of Enterococcus faecalis in root canals ex vivo. Int Endod J 2005;38:735-742.

23. Gomes BP, Montagner F, Jacinto RC, Zaia AA, Ferraz CC, Souza-Filho FJ. Polymerase chain reaction of porphyromonas gingivalis, treponema denticola, and tannerella forsythia in primary endodontic infections. J Endod 2007;33:1049-1052.

24. Rocas IN, Siqueira JF, Santos KRN, Coelho AMA. 'Red complex' (bacteroides forsythus, porphyromonas gingivalis, and treponema denticola) in endodontic infections: a molecular approach. Oral Surg Oral Med Oral Pathol Oral Radiol Endod 2001;91(4):468-471.

25. Rupf S, Kannengiesser S, Merte K, Pfister W, Sigusch B, Eschrich K. Comparison of profiles of key periodontal pathogens in periodontium and endodontium. Endod Dent Traumatol 2000;16:269-275.

26. Li H, Guan R, Sun J, Hou B. Bacteria community study of combined periodontal-endodontic lesions using denaturing gradient gel electrophoresis and sequencing analysis. J Periodontol 2014;85:1442-1449.

27. Nair PNR. Light and electron microscopic studies of root canal flora and periapical lesions. J Endod 1987;13:29-39.
28. Costerton W, Veeh R, Shirtliff M, Pasmore M, Post C, Ehrlich $\mathrm{G}$. The application of biofilm science to the study and control of chronic bacterial infections. J Clin Invest 2003;112: 1466-1477.

29. Johnson BT, Shaw LN, Nelson DC, Mayo JA. Extracellular proteolytic activities expressed by Bacillus pumilus isolated from endodontic and periodontal lesions. J Med Microbiol 2008 Feb;57:643-651.

30. Minghui Xia, Qingguo Qi. Bacterial analysis of combined periodontal-endodontic lesions by polymerase chain reaction denaturing gradient gel electrophoresis. J Oral Sci 2013;55(4): 287-291.

31. Pereira CV, Stipp RN, Fonseca DC, Pereira LJ, Hofling JF. Detection and clonal analysis of anaerobic bacteria associated to endodontic-periodontal lesions. J Periodontol 2011;82: 1767-1775.

32. Contreras A, Umeda M, Chen C, Bakker I, Morrison JL, Slots $\mathrm{J}$. Relationship between herpes viruses and adult periodontitis and periodontopathic bacteria. J Periodontol 1999;70: 478-484. 